\title{
Biomechanical Simulation of Electrode Migration for Deep Brain Stimulation
}

\author{
Alexandre Bilger, Jérémie Dequidt, Christian Duriez, and Stéphane Cotin \\ SHAMAN Group, INRIA Lille North Europe
}

\begin{abstract}
Deep Brain Stimulation is a modern surgical technique for treating patients who suffer from affective or motion disorders such as Parkinson's disease. The efficiency of the procedure relies heavily on the accuracy of the placement of a micro-electrode which sends electrical pulses to a specific part of the brain that controls motion and affective symptoms. However, targeting this small anatomical structure is rendered difficult due to a series of brain shifts that take place during and after the procedure. This paper introduces a biomechanical simulation of the intra and postoperative stages of the procedure in order to determine lead deformation and electrode migration due to brain shift. To achieve this goal, we propose a global approach, which accounts for brain deformation but also for the numerous interactions that take place during the procedure (contacts between the brain and the inner part of the skull and falx cerebri, effect of the cerebro-spinal fluid, and biomechanical interactions between the brain and the electrodes and cannula used during the procedure). Preliminary results show a good correlation between our simulations and various results reported in the literature.
\end{abstract}

\section{Introduction}

Deep Brain Stimulation (DBS) is a modern surgical treatment of brain disorders such as Parkinson's disease or dystonia. This procedure consists in the placement of a micro-electrode in the subthalamic area, deep into the brain. The placement of the electrode is crucial to maximize outcomes and to prevent adverse effects. This placement is achieved in two main stages: first, pre-operative medical images of the patient are combined with the use of a stereotactic frame (and sometimes an atlas of the brain) to determine the target coordinates and optimal trajectory for the electrode(s). Second, the patient is taken to the operating room where a macro-electrode attached to a thin wire is inserted into the brain according to the planned trajectory. However, a combination of brain shift and a certain inaccuracy in the exact location of the target area require to test the area next to the planned target to optimize the placement of the electrode. This testing is performed by recording the brain activity using the macro-electrode. This entire process takes several hours, and once the appropriate area has been identified the final (micro) electrode will be left in place and secured to the skull. However, two major problems arise during this process: first, the planning stage does not account for the brain shift that takes place during surgery. Depending on

G. Fichtinger, A. Martel, and T. Peters (Eds.): MICCAI 2011, Part I, LNCS 6891, pp. 339-346, 2011.

(C) Springer-Verlag Berlin Heidelberg 2011 
the amplitude of the brain shift, the effective location for the electrode can be quite remote from the planned location ( $5 \mathrm{~mm}$ or more) [13] requiring extensive mapping of the area to determine the final location. As a consequence, this stage of the procedure usually lasts several hours, while the patient is awake, and increases the likelihood of complications due to the number of insertions to reach the targeted area 4 . The second problem, also linked to the amplitude of the brain shift, takes places several days or weeks after the surgery. As reported in [13. a post-operative electrode displacement and deformation may appear as the brain returns to its initial position when the subdural air introduced during surgery has resolved (see Fig,1). This hinders the efficiency of the procedure because upward migration of the electrode may fail to correctly stimulate the subthalamic area.

In this context, our objective is to propose a global approach that can model these two phenomena in order to adjust the planned trajectory, determine a potential post-operative electrode migration, and propose alternative strategies to minimize its amplitude. As a first step in this direction, this paper introduces an original and unified approach to model the brain behavior during a DBS procedure. The focus of the paper is not set on a specific biomechanical model of the brain but rather on a complete framework that is able to simulate intra-cranial fluid loss, subdural air invasion, brain shift and electrode migration and curvature. This work includes the following contributions: mechanical models of the brain and the devices (cannula and electrode); mechanical interactions between the brain and these devices (in particular electrode deformation when the brain reverts to its initial shape); influence of the surrounding cerebro-spinal fluid (CSF) and air invasion in the skull on the brain shift (including the asymmetry of the brain shift). Results of the simulation exhibit qualitatively consistent results compared to these various points reported in the literature.

This paper is organized as follows: section 2 reviews previous works related to simulation of brain and DBS, section 3 details the various elements of our approach, and finally section 4 provides preliminary results.
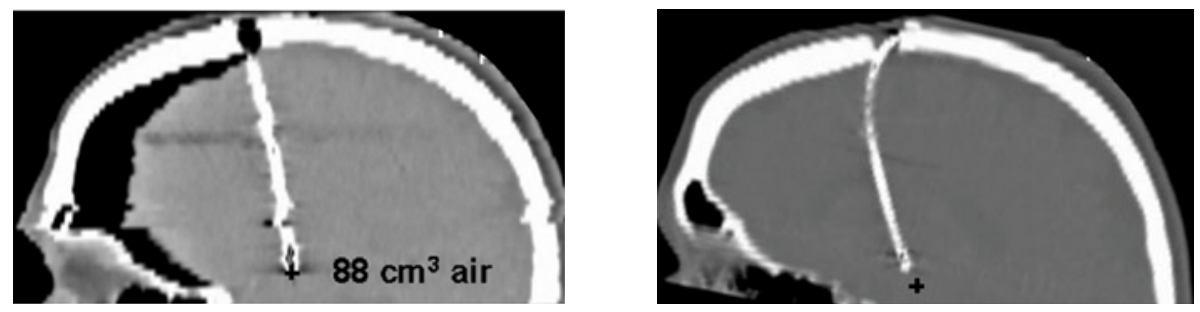

Fig. 1. Post-operative (left) and follow-up (right) CT scans. The post-operative scan illustrates the brain shift at the end of the procedure. The follow-up scan emphasizes the deformation of the electrode due to the inverse brain shift, leading to an upward migration of the electrode away from its initial location after the craniotomy (black cross). For large brain shifts, the electrode can move of up to $5 \mathrm{~mm}$. Courtesy of [13. 


\section{Previous Works}

As stated in the introduction, the craniotomy-induced brain shift is one of the main factors that impact the planning, duration and complexity of DBS procedures. Brain shift also impacts many other neurosurgical procedures. In the literature related to brain shift, the focus is on measuring or computing the displacement field of the brain in order to accurately track the targeted area (for instance a tumor). Most of these approaches rely on more or less advanced biomechanical models of the brain, usually guided by coarse intra-operative information such as ultrasonography 2, intra-operative MRI 3] or laser-range scanner [15. However, such approaches, that typically use per-operative information to post-correct the deformation of the brain to match measured surface displacement rely on oversimplified boundary conditions. These approaches fail to correctly reproduce the complex interactions that take place inside the skull. While this is acceptable when only local tissue deformation is sought (e.g. tumor removal near the brain surface), this becomes a limitation for a more global estimation of the deformation (as needed for DBS). They also do not consider post-operative recovery of the brain which plays a crucial role in deep brain stimulations because of possible electrode migration, which has been recently highlighted by [6]13.

Estimating the final position (e.g. after brain recovery) of the electrode requires to take into account various elements in the simulation: a) biomechanical brain model, b) bilateral and unilateral boundary conditions with the skull and falx cerebri, c) interactions between the brain, the cannula and the electrode(and its wire) and finally d) loss of CSF due to the craniotomy. To our knowledge, such a complete and unified framework has not been addressed in the literature. Some works have proposed advanced biomechanical brain models based on linear elasticity [3] or non-linear visco-elasticity [11]. The influence of boundary conditions is emphasized in [14] where three scenarios for brain-skull boundary conditions are compared and experiments show that best results are achieved when brain motion is allowed in the cranial cavity. Regarding the insertion of the cannula or electrode in the brain, the closest works are in the field of brachytherapy (the reader may refer to [1] for a survey on the insertion of needles into soft tissues). Finally regarding the influence of CSF, Lunn et al $[8]$ propose to model the brain as a porous media and use consolidation theory to take into account the CSF in the brain.

\section{Simulation Framework and Methods}

The principal contribution of this paper is to propose a global, physics-based approach that models the main (bio)mechanical phenomena that can be observed during and after a DBS procedure, i.e.:

- Asymmetric brain shift which can be observed during the first electrode implantation. It is due to a unilateral air invasion on the side of the first craniotomy, resulting in a contralateral brain shift. 
- Two-stage brain shift which appears after removing the cannula. While most of the brain shift takes place right after the craniotomy and before the insertion of the cannula and macro-electrode, the air invasion continues to occur during the lengthy testing process. However, the cannula being rigid, it locally reduces brain shift. When the cannula is removed, an additional shift of the brain can sometimes be observed and can drive to a first deformation of the flexible electrode.

- Inverse brain shift which takes place several days or weeks after the procedure. As the air invasion is resolved and new CSF has been produced, the brain recovers its initial shape and position. During this inverse brain shift, a deformation is also applied to the micro-electrode and its wire. This interaction creates a relative motion between the electrode and the brain, along the axis of the electrode. If this motion is important, the electrodes are no longer able to stimulate the correct subthalamic area.

\subsection{Cerebro-Spinal Fluid Model}

Brain shift takes place due to a loss of cerebro-spinal fluid and subdural air invasion after the skull opening(see Fig 3). Before the craniotomy, the buoyancy force created by the CSF balances the gravity force acting on the brain. But after a loss of fluid through the burr hole, a part of the cerebral tissue is left above the remaining CSF surface and undergoes only gravity. This unbalance can deform and move the brain significantly. Reported measures indicate that even deep brain structures (e.g. the targets of a DBS such as the Subthalamic Nucleus) may shift of up to $5 \mathrm{~mm} 12$.

The external force created on the surface of the brain by the CSF is computed as $\mathbf{f}_{C S F}=\iint_{S} \rho g h(P) \mathrm{d} \mathbf{S}$ with $\rho$ is the density of CSF, $g$ the norm of the gravity and $h$ the distance between a point $\mathrm{P}$ on the brain surface and the fluid level. This force is computed on each triangle $\mathrm{S}$ of the brain mesh (see below) that corresponds to the immersed surface. Results are illustrated in section 4 .

\subsection{Constrained Deformations of the Brain}

The anatomy of the brain and skull is based on a generic atlas and is not issued from patient data. The deformations of the brain are modeled using the finite element method. The volume of the brain hemispheres is meshed as a set of tetrahedral elements, and different constitutive models can be used to describe the brain deformation. In the current results, we have used both a co-rotational approach (to capture the geometrical non-linearities of the deformations) and a Saint Venant-Kirchhoff model to describe a hyperelastic material. While there are other constitutive laws that have been proposed for modeling brain tissue, we want to emphasize in this section the importance of correctly accounting for the complex boundary conditions, which can have a larger impact on the final deformation than the choice of a particular deformation model. In the particular case of the CSF influence on brain deformation, the change of forces acting on the brain leads to a brain shift, while the falx cerebri which separates the two 

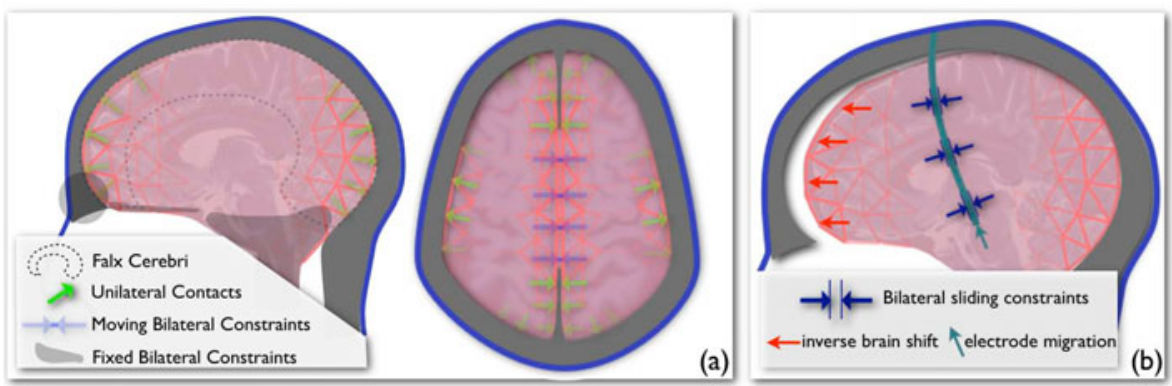

Fig. 2. Illustration of the different models used by the simulation and their relative boundary conditions. The use of complex constraints, combined with a FEM modeling technique permits to capture some of the key characteristics of a DBS procedure.

brain hemispheres limits the influence of the CSF loss and air invasion to the side of the craniotomy. This relatively complex process is simulated by modeling independently each hemisphere of the brain and by precisely defining boundary conditions between the brain hemispheres themselves, and between the brain and skull or falx cerebri.

Independently of the choice of the deformation model, we end up with the following differential system of non-linear equations $\mathbf{M a}=\mathbf{f}(\mathbf{x}, \mathbf{v})+\mathbf{p}+\mathbf{f}_{C S F}+\mathbf{H}^{T} \lambda$ where $\mathbf{M}$ is the mass matrix, $\mathbf{f}$ gathers the internal forces. $\mathbf{a}, \mathbf{v}$ and $\mathbf{x}$ are respectively the acceleration, the velocity and the position of the nodes from the mesh. The forces $\mathbf{p}$ are exerted by the gravity and $\mathbf{f}_{C S F}$ by the CSF. Finally, $\mathbf{H}^{T} \lambda$ gathers constraints response resulting from unilateral contacts and bilateral constraints (e.g. see Fig. 2 for brain-falx cerebri and brain-skull contacts; moving bilateral constraints between the two brain hemispheres; fixed bilateral constraints near the area of the optic nerves and the brainsterm). An implicit integration scheme (Backward Euler) is used to enforce stability even when using large time steps.

\subsection{Brain-Electrode Interactions}

With the objective to estimate the relative motion between the electrode and the brain during the inverse brain shift, and define new strategies to compensate for that, it is essential to accurately model the mechanical interactions between the cannula, the electrode and the brain tissue during the different stages of the insertion and deformation. The electrode and cannula are modeled using seriallylinked beams elements as done in [5] for coil modeling. This non-linear elastic model can be parametrized to reproduce the resistance to bending, stretching and torsion of the two types of devices. The mechanical coupling between the devices and brain is controlled using constraints that are solved using additional Lagrange Multipliers, following the approach presented in [5]. A set of sliding point constraints is positioned in the brain model along the path of the cannula; and a similar set of constraints is used for the electrode. For solving the coupling 


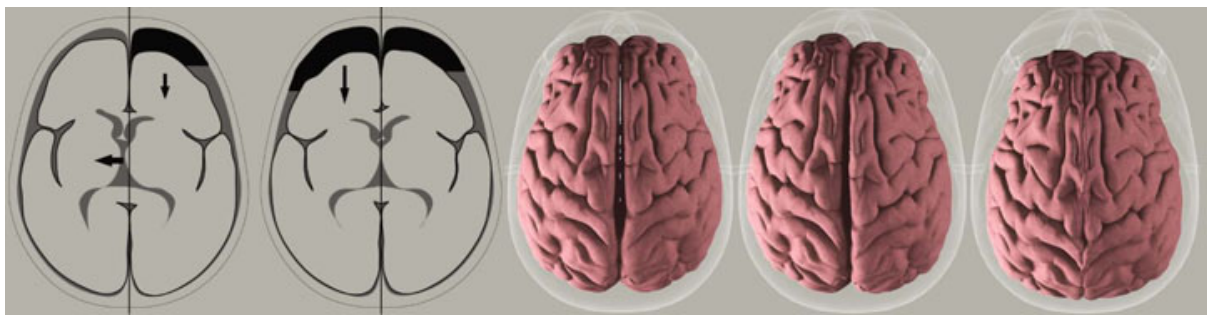

Fig. 3. Asymmetrical brain shift simulation: the brain shift is more important on the side where the craniotomy first takes place, as illustrated in the left most images (courtesy of 9]). The rightmost images show the evolution of brain shift during our simulation: (a) no brain shift; (b) after a right craniotomy; and (c) after both craniotomies.

between the models, Lagrange Multipliers are set for both the brain tissue model and beam models. The final values of the Lagrange Multipliers are solved using a Mixed-Complementarity Problem solver (MCP).

\section{Results}

In this section we present a series of results for the main steps of the procedure described in section 3. The conditions and parameters used in the simulation reproduce as closely as possible the conditions and parameters reported in the literature to facilitate comparisons, i.e : the patient lies in the supine position with the head elevated at 10 to 20 degrees; the craniotomy is performed first on the right side of the skull; a first brain shift takes place before the cannula is inserted through the right hemisphere; the electrode and wire are then inserted through the cannula; the cannula is removed and the electrode remains in the brain but is slightly shifted due to secondary brain shift; the CSF is restored and the air is removed to simulate post-operative conditions several weeks after the surgery. The same process is repeated for the left side of the brain. As values reported in the literature for the Young's modulus range from 2,100 [10] to $40,000 \mathrm{~Pa}$ [7, we use, for both the co-rotational and Saint Venant Kirchhoff models a Young's modulus $E=6000 P a$ and a Poisson ratio $\nu=0.45$ for a total mass of the brain of $1.4 \mathrm{~kg}$. The density of CSF $\rho$ is set to $1000 \mathrm{~kg} / \mathrm{m}^{3}$. Figure 3 illustrates the simulation of the asymmetric brain shift. During the surgery and even after the loss of CSF, the brain shifts continuously because of the pressure due to air invasion. However, the rigid material of the cannula prevents the brain motion. That is why, the removal of the cannula causes a second but minor brain shift. This effect involves an anteroposterior deformation of the implanted wire, as depicted in figure 4. Several days after surgery no more air is in the cranial space and the CSF has been restored. We simulate this effect by modifying the CSF level. The resulting deformations of the brain are computed using our FEM approach. As the electrode and its wire are constrained within the brain (only sliding is possible) and the wire is secured on the skull surface, this results in a 

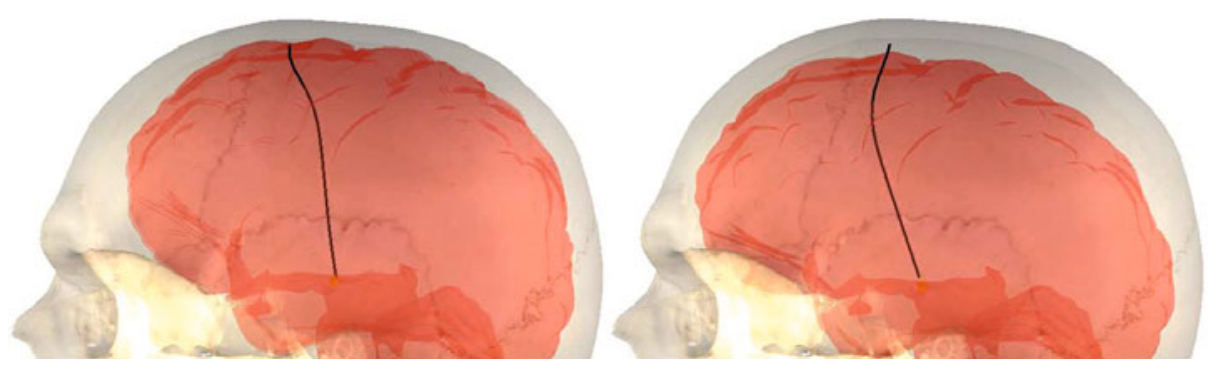

Fig. 4. Screenshot showing the deflection of the right electrode after the cannula removal (left) and after CSF recovery (right).

relative motion of the electrode with respect to its intra-operative location. This leads to a posteroanterior curvature, as illustrate in figure 4 which correlates very well with data from figure 1.

We also quantitatively compared our results (amplitude of electrode migration) with data reported in 13 . In their study, a correlation between the volume of subdural air and upward electrode displacement along its trajectory was determined. The relationship between the displacement $D$ (in $\mathrm{mm}$ ) and subdural air volume $V$ (in $\mathrm{cm}^{3} 3$ ) can be empirically described as $D=2+0.08 * V$. We simulated two relatively different amounts of CSF loss, and computed the corresponding values of $V$ (as the difference between the volume of the brain before and after brain shift) and $D$. We found that for $V=22 \mathrm{~cm}^{3}$ a displacement $D=3 \mathrm{~mm}$ is computed, compared to $3.7 \mathrm{~mm} \pm 2 \mathrm{~mm}$ according to the experimental data [13. Similarly, for $V=62 \mathrm{~cm}^{3}$ a displacement $D=6.8 \mathrm{~mm}$ is computed, compared to $D=6.96 \mathrm{~mm} \pm 2.5 \mathrm{~mm}$ according to the experimental law above. This strong correlation between our results and published data illustrates the potential of our method. It is also important to note that the entire simulation of the combined models (from pre- to post-operative stages) only requires a few minutes to be computed.

\section{Conclusion}

Preliminary results show a good correlation with data reported in the literature, while all our results are obtained using a unique physics-based framework that offers a global approach rather than independently modeling each phenomena. In addition, we account for the interaction between the brain and electrode, and show that our simulations quantitatively correlate with recently reported experimental data. Finally, our computation times remain compatible with future clinical use. Obviously, this is only a first step, and the accuracy of our simulations could certainly be increased by integrating feedback from intraoperative data in the form of additional constraints. This is our next objective, which will also facilitate direct comparison with intra and post-operative results. 
Acknowledegment. The authors would like to thank the French Research Agency (ANR) for funding this study through the ACouStiC project.

\section{References}

1. Abolhassani, N., Patel, R., Moallem, M.: Needle insertion into soft tissue: A survey. Medical Engineering \& Physics 29(4), 413 (2007)

2. Bucki, M., Lobos, C., Payan, Y.: Framework for a low-cost intra-operative imageguided neuronavigator including brain shift compensation. In: IEEE Engineering in Medicine and Biology Society, pp. 872-875 (2007)

3. Clatz, O., Delingette, H., Talos, I.F., Golby, A.J., Kikinis, R., Jolesz, F.A., Ayache, N., Warfield, S.K.: Robust nonrigid registration to capture brain shift from intraoperative mri. IEEE Transactions on Medical Imaging 24(11), 1417-1427 (2005)

4. Deep-Brain Stimulation for Parkinson's Disease Study Group: Deep-brain stimulation of the subthalamic nucleus or the pars interna of the globus pallidus in parkinson's disease. N. Engl. J. Med. 345(13), 956-963 (2001)

5. Duriez, C., Guébert, C., Marchal, M., Cotin, S., Grisoni, L.: Interactive simulation of flexible needle insertions based on constraint models. In: Yang, G.-Z., Hawkes, D., Rueckert, D., Noble, A., Taylor, C. (eds.) MICCAI 2009. LNCS, vol. 5762, pp. 291-299. Springer, Heidelberg (2009)

6. Kim, Y.H., Kim, H.J., Kim, C., Kim, D.G., Jeon, B.S., Paek, S.H.: Comparison of electrode location between immediate postoperative day and 6 months after bilateral subthalamic nucleus stimulation. Acta Neurochir 152(12), 2037-2045 (2010)

7. Kruse, S.A., Rose, G.H., Glaser, K.J., Manduca, A., Felmlee, J.P., Jack Jr., C.R., Ehman, R.L.: Magnetic resonance elastography of the brain. NeuroImage 39(1), 231-237 (2008)

8. Lunn, K.E., Paulsen, K.D., Lynch, D.R., Roberts, D.W., Kennedy, F.E., Hartov, A.: Assimilating intraoperative data with brain shift modeling using the adjoint equations. Medical Image Analysis 9(3), 281-293 (2005)

9. Lurig, C., Hastreiter, P., Nimsky, C., Ertl, T.: Analysis and visualization of the brain shift phenomenon in neurosurgery. In: TCVG Symposium on Visualization (VisSym), pp. 285-290 (1999)

10. Miga, M., Paulsen, K., Hoopes, P., Kennedy Jr., F., Hartov, A., Roberts, D.: In vivo quantification of a homogeneous brain deformation model for updating preoperative images during surgery. Biomedical Engineering 47(2), 266-273 (2000)

11. Miller, K., Wittek, A., Joldes, G.: Biomechanics of the brain for computerintegrated surgery. publishing House of Warsaw University of Technology (2002)

12. Miyagi, Y., Shima, F., Sasaki, T.: Brain shift: an error factor during implantation of deep brain stimulation electrodes. Journal of Neurosurgery 107(5), 989-997 (2007)

13. Munckhof, P.V.D., Contarino, M.F., Bour, L.J., Speelman, J.D., Bie, R.M.A.D., Schuurman, P.R.: Postoperative curving and upward displacement of deep brain stimulation electrodes caused by brain shift. Journal of Neurosurgery 67(1), 49-54 (2010)

14. Wittek, A., Miller, K., Kikinis, R., Warfield, S.K.: Patient-specific model of brain deformation: Application to medical image registration. Journal of Biomechanics 40(4), 919-929 (2007)

15. Zhang, C., Wang, M., Song, Z.: A brain-deformation framework based on a linear elastic model and evaluation using clinical data. Transactions on Biomedical Engineering 58(1), 1-9 (2011) 